\title{
COMPLEX CONNECTIONS ON CONFORMAL KÄHLER MANIFOLDS WITH NORDEN METRIC
}

\author{
MARTA TEOFILOVA
}

\begin{abstract}
An eight-parametric family of complex connections on a class complex manifolds with Norden metric is introduced. The form of the curvature tensor with respect to each of these connections is obtained. The conformal group of the considered connections is studied and some conformal invariants are obtained.

2010 Mathematics Subject Classification: 53C15, 53C50.

Keywords: complex connection, complex manifold, Norden metric.
\end{abstract}

\section{INTRODUCTION}

Almost complex manifolds with Norden metric were first studied by A. P. Norden [9] and are introduced in [4] as generalized $B$-manifolds. A classification of these manifolds with respect to the covariant derivative of the almost complex structure is obtained in [1] and two equivalent classifications are given in [2, 3.

An important problem in the geometry of almost complex manifolds with Norden metric is the study of linear connections preserving the almost complex structure or preserving both, the structure and the metric. The first ones are called almost complex connections, and the second ones are known as natural connections. A special type of natural connection is the canonical one. In [2] it is proved that on an almost complex manifold with Norden metric there exists a unique canonical connection. The canonical connection (called also the $B$-connection) and its conformal group on a conformal Kähler manifold with Norden metric are studied in 3.

In [11] we have obtained a two-parametric family of complex connections on a conformal Kähler manifold with Norden metric and have proved that the curvature tensors corresponding to these connections coincide with the curvature tensor of the canonical connection.

In the present work we continue our research on complex connections on complex manifolds with Norden metric by focusing our attention on the class of the conformal Kähler manifolds, i.e. manifolds which are conformally equivalent to Käher manifolds with Norden metric. We introduce an eight-parametric family of complex connections on such manifolds and consider their curvature properties. We also study the conformal group of these connections and obtain some conformal invariants. In the last section we give an example of a four-dimensional conformal Kähler manifold with Norden metric, on which the considered complex connections are flat. 


\section{Preliminaries}

Let $(M, J, g)$ be a $2 n$-dimensional almost complex manifold with Norden metric, i.e. $J$ is an almost complex structure and $g$ is a pseudo-Riemannian metric on $M$ such that

$$
J^{2} x=-x, \quad g(J x, J y)=-g(x, y)
$$

for all differentiable vector fields $x, y$ on $M$, i.e. $x, y \in \mathfrak{X}(M)$.

The associated metric $\widetilde{g}$ of $g$ is given by $\widetilde{g}(x, y)=g(x, J y)$ and is a Norden metric, too. Both metrics are necessarily neutral, i.e. of signature $(n, n)$.

If $\nabla$ is the Levi-Civita connection of the metric $g$, the fundamental tensor field $F$ of type $(0,3)$ on $M$ is defined by

$$
F(x, y, z)=g\left(\left(\nabla_{x} J\right) y, z\right)
$$

and has the following symmetries

$$
F(x, y, z)=F(x, z, y)=F(x, J y, J z) .
$$

Let $\left\{e_{i}\right\}(i=1,2, \ldots, 2 n)$ be an arbitrary basis of $T_{p} M$ at a point $p$ of $M$. The components of the inverse matrix of $g$ are denoted by $g^{i j}$ with respect to the basis $\left\{e_{i}\right\}$. The Lie 1 -forms $\theta$ and $\theta^{*}$ associated with $F$, and the Lie vector $\Omega$, corresponding to $\theta$, are defined by, respectively

$$
\theta(x)=g^{i j} F\left(e_{i}, e_{j}, x\right), \quad \theta^{*}=\theta \circ J, \quad \theta(x)=g(x, \Omega) .
$$

The Nijenhuis tensor field $N$ for $J$ is given by [7]

$$
N(x, y)=[J x, J y]-[x, y]-J[J x, y]-J[x, J y] .
$$

It is known [8] that the almost complex structure is complex if and only if it is integrable, i.e. iff $N=0$.

A classification of the almost complex manifolds with Norden metric is introduced in [1, where eight classes of these manifolds are characterized according to the properties of $F$. The three basic classes $\mathcal{W}_{i}(i=1,2,3)$ are given by

- the class $\mathcal{W}_{1}$ :

$$
\begin{aligned}
F(x, y, z)=\frac{1}{2 n}[ & g(x, y) \theta(z)+g(x, J y) \theta(J z) \\
& +g(x, z) \theta(y)+g(x, J z) \theta(J y)] ;
\end{aligned}
$$

- the class $\mathcal{W}_{2}$ of the special complex manifolds with Norden metric:

$$
F(x, y, J z)+F(y, z, J x)+F(z, x, J y)=0, \quad \theta=0 \quad \Leftrightarrow \quad N=0, \quad \theta=0 ;
$$

- the class $\mathcal{W}_{3}$ of the quasi-Kähler manifolds with Norden metric:

$$
F(x, y, z)+F(y, z, x)+F(z, x, y)=0 .
$$

The special class $\mathcal{W}_{0}$ of the Kähler manifolds with Norden metric is characterized by the condition $F=0(\nabla J=0)$ and is contained in each one of the other classes.

Let $R$ be the curvature tensor of $\nabla$, i.e. $R(x, y) z=\nabla_{x} \nabla_{y} z-\nabla_{y} \nabla_{x} z-\nabla_{[x, y]} z$. The corresponding (0,4)-type tensor is defined by $R(x, y, z, u)=g(R(x, y) z, u)$.

A tensor $L$ of type $(0,4)$ is called a curvature-like tensor if it has the properties of $R$, i.e.

$$
\begin{aligned}
& L(x, y, z, u)=-L(y, x, z, u)=-L(x, y, u, z) \\
& L(x, y, z, u)+L(y, z, x, u)+L(z, x, y, u)=0 .
\end{aligned}
$$


The Ricci tensor $\rho(L)$ and the scalar curvatures $\tau(L)$ and $\tau^{*}(L)$ of $L$ are defined by:

$$
\begin{gathered}
\rho(L)(y, z)=g^{i j} L\left(e_{i}, y, z, e_{j}\right), \\
\tau(L)=g^{i j} \rho(L)\left(e_{i}, e_{j}\right), \quad \tau^{*}(L)=g^{i j} \rho(L)\left(e_{i}, J e_{j}\right) .
\end{gathered}
$$

A curvature-like tensor $L$ is called a Kähler tensor if

$$
L(x, y, J z, J u)=-L(x, y, z, u) .
$$

Let $S$ be a tensor of type $(0,2)$. We consider the following tensors [3]:

$$
\begin{aligned}
& \psi_{1}(S)(x, y, z, u)=g(y, z) S(x, u)-g(x, z) S(y, u) \\
&+g(x, u) S(y, z)-g(y, u) S(x, z), \\
& \psi_{2}(S)(x, y, z, u)= g(y, J z) S(x, J u)-g(x, J z) S(y, J u) \\
&+ g(x, J u) S(y, J z)-g(y, J u) S(x, J z), \\
& \pi_{1}=\frac{1}{2} \psi_{1}(g), \quad \pi_{2}=\frac{1}{2} \psi_{2}(g), \quad \pi_{3}=-\psi_{1}(\widetilde{g})=\psi_{2}(\widetilde{g}) .
\end{aligned}
$$

The tensor $\psi_{1}(S)$ is curvature-like if $S$ is symmetric, and the tensor $\psi_{2}(S)$ is curvature-like if $S$ is symmetric and hybrid with respect to $J$, i.e. $S(x, J y)=$ $S(y, J x)$. In the last case the tensor $\left\{\psi_{1}-\psi_{2}\right\}(S)$ is Kählerian. The tensors $\pi_{1}-\pi_{2}$ and $\pi_{3}$ are also Kählerian.

The usual conformal transformation of the Norden metric $g$ (conformal transformation of type I [3]) is defined by

$$
\bar{g}=e^{2 u} g,
$$

where $u$ is a pluriharmonic function, i.e. the 1 -form $d u \circ J$ is closed.

A $\mathcal{W}_{1}$-manifold with closed 1 -forms $\theta$ and $\theta^{*}$ (i.e. $\mathrm{d} \theta=\mathrm{d} \theta^{*}=0$ ) is called a conformal Kähler manifold with Norden metric. Necessary and sufficient conditions for a $\mathcal{W}_{1}$-manifold to be conformal Kählerian are:

$$
\left(\nabla_{x} \theta\right) y=\left(\nabla_{y} \theta\right) x, \quad\left(\nabla_{x} \theta\right) J y=\left(\nabla_{y} \theta\right) J x .
$$

The subclass of these manifolds is denoted by $\mathcal{W}_{1}^{0}$.

It is proved $\left[3\right.$ that a $\mathcal{W}_{1}^{0}$-manifold is conformally equivalent to a Kähler manifold with Norden metric by the transformation (2.12).

It is known that on a pseudo-Riemannian manifold $M(\operatorname{dim} M=2 n \geq 4)$ the conformal invariant Weyl tensor has the form

$$
W(R)=R-\frac{1}{2(n-1)}\left\{\psi_{1}(\rho)-\frac{\tau}{2 n-1} \pi_{1}\right\} .
$$

Let $L$ be a Kähler curvature-like tensor on an almost complex manifold with Norden metric $(M, J, g), \operatorname{dim} M=2 n \geq 6$. Then the Bochner tensor $B(L)$ for $L$ is defined by [3]:

$$
\begin{aligned}
B(L) & =L-\frac{1}{2(n-2)}\left\{\psi_{1}-\psi_{2}\right\}(\rho(L)) \\
& +\frac{1}{4(n-1)(n-2)}\left\{\tau(L)\left(\pi_{1}-\pi_{2}\right)+\tau^{*}(L) \pi_{3}\right\} .
\end{aligned}
$$

\section{Complex Connections on $\mathcal{W}_{1}$-Manifolds}

Definition 3.1 ([7]). A linear connection $\nabla^{\prime}$ on an almost complex manifold $(M, J)$ is said to be almost complex if $\nabla^{\prime} J=0$.

We introduce an eight-parametric family of complex connections in the following 
Theorem 3.1. On a $\mathcal{W}_{1}$-manifold with Norden metric there exists an eight-parametric family of complex connections $\nabla^{\prime}$ defined by

$$
\nabla_{x}^{\prime} y=\nabla_{x} y+Q(x, y)
$$

where the deformation tensor $Q(x, y)$ is given by

$$
\begin{aligned}
Q(x, y)= & \frac{1}{2 n}[\theta(J y) x-g(x, y) J \Omega] \\
& +\frac{1}{n}\left\{\lambda_{1} \theta(x) y+\lambda_{2} \theta(x) J y+\lambda_{3} \theta(J x) y+\lambda_{4} \theta(J x) J y\right. \\
& +\lambda_{5}[\theta(y) x-\theta(J y) J x]+\lambda_{6}[\theta(y) J x+\theta(J y) x] \\
& \left.+\lambda_{7}[g(x, y) \Omega-g(x, J y) J \Omega]+\lambda_{8}[g(x, J y) \Omega+g(x, y) J \Omega]\right\},
\end{aligned}
$$

$\lambda_{i} \in \mathbb{R}, i=1,2, \ldots, 8$.

Proof. By (2.5), (3.1) and (3.2) we verify that $\left(\nabla_{x}^{\prime} J\right) y=\nabla_{x}^{\prime} J y-J \nabla_{x}^{\prime} y=0$, and hence the connections $\nabla^{\prime}$ are complex for any $\lambda_{i} \in \mathbb{R}, i=1,2, \ldots, 8$.

Let us remark that the two-parametric family of complex connections obtained for $\lambda_{1}=\lambda_{4}, \lambda_{3}=-\lambda_{2}, \lambda_{5}=\lambda_{7}=0, \lambda_{8}=-\lambda_{6}=\frac{1}{4}$, is studied in [11.

Let $T^{\prime}$ be the torsion tensor of $\nabla^{\prime}$, i.e. $T^{\prime}(x, y)=\nabla_{x}^{\prime} y-\nabla_{x}^{\prime} y-[x, y]$. Taking into account that the Levi-Civita connection $\nabla$ is symmetric, we have $T^{\prime}(x, y)=$ $Q(x, y)-Q(y, x)$. Then by (3.2) we obtain

$$
\begin{aligned}
T^{\prime}(x, y) & =\frac{1}{n}\left\{\left(\lambda_{1}-\lambda_{5}\right)[\theta(x) y-\theta(y) x]+\left(\lambda_{2}-\lambda_{6}\right)[\theta(x) J y-\theta(y) J x]\right. \\
& \left.+\left(\lambda_{3}-\lambda_{6}-\frac{1}{2}\right)[\theta(J x) y-\theta(J y) x]+\left(\lambda_{4}+\lambda_{5}\right)[\theta(J x) J y-\theta(J y) J x]\right\} .
\end{aligned}
$$

It is easy to verify the following

$$
\underset{x, y, z}{\mathfrak{S}} T^{\prime}(x, y, z)=\underset{x, y, z}{\mathfrak{S}} T^{\prime}(J x, J y, z)=\underset{x, y, z}{\mathfrak{S}} T^{\prime}(x, y, J z)=0,
$$

where $\mathfrak{S}$ is the cyclic sum by the arguments $x, y, z$.

Next, we obtain necessary and sufficient conditions for the complex connections $\nabla^{\prime}$ to be symmetric (i.e. $T^{\prime}=0$ ).

Theorem 3.2. The complex connections $\nabla^{\prime}$ defined by (3.1) and (3.2) are symmetric on a $\mathcal{W}_{1}$-manifold with Norden metric if and only if $\lambda_{1}=-\lambda_{4}=\lambda_{5}$, $\lambda_{2}=\lambda_{3}-\frac{1}{2}=\lambda_{6}$.

Then, by putting $\lambda_{1}=-\lambda_{4}=\lambda_{5}=\mu_{1}, \lambda_{2}=\lambda_{6}=\lambda_{3}-\frac{1}{2}=\mu_{2}, \lambda_{7}=\mu_{3}, \lambda_{8}=\mu_{4}$ in (3.2) we obtain a four-parametric family of complex symmetric connections $\nabla^{\prime \prime}$ on a $\mathcal{W}_{1}$-manifold which are defined by

$$
\begin{aligned}
\nabla_{x}^{\prime \prime} y & =\nabla_{x} y+\frac{1}{2 n}[\theta(J x) y+\theta(J y) x-g(x, y) J \Omega] \\
& +\frac{1}{n}\left\{\mu_{1}[\theta(x) y+\theta(y) x-\theta(J x) J y-\theta(J y) J x]\right. \\
& +\mu_{2}[\theta(J x) y+\theta(J y) x+\theta(x) J y+\theta(y) J x] \\
& \left.+\mu_{3}[g(x, y) \Omega-g(x, J y) J \Omega]+\mu_{4}[g(x, J y) \Omega+g(x, y) J \Omega]\right\} .
\end{aligned}
$$

The well-known Yano connection [12, 13, on a $\mathcal{W}_{1}$-manifold is obtained from (3.5) for $\mu_{1}=\mu_{3}=0, \mu_{4}=-\mu_{2}=\frac{1}{4}$.

Definition $3.2([2])$. A linear connection $\nabla^{\prime}$ on an almost complex manifold with Norden metric $(M, J, g)$ is said to be natural if $\nabla^{\prime} J=\nabla^{\prime} g=0$ (or equivalently, $\left.\nabla^{\prime} g=\nabla^{\prime} \widetilde{g}=0\right)$. 
From (3.1) and (3.2) we compute the covariant derivatives of $g$ and $\widetilde{g}$ with respect to the complex connections $\nabla^{\prime}$ as follows

$$
\begin{aligned}
& \left(\nabla_{x}^{\prime} g\right)(y, z)=-Q(x, y, z)-Q(x, z, y) \\
& =-\frac{1}{n}\left\{2 \left[\lambda_{1} \theta(x) g(y, z)+\lambda_{2} \theta(x) g(y, J z)+\lambda_{3} \theta(J x) g(y, z)\right.\right. \\
& \left.+\lambda_{4} \theta(J x) g(y, J z)\right]+\left(\lambda_{5}+\lambda_{7}\right)[\theta(y) g(x, z)+\theta(z) g(x, y) \\
& -\theta(J y) g(x, J z)-\theta(J z) g(x, J y)]+\left(\lambda_{6}+\lambda_{8}\right)[\theta(y) g(x, J z) \\
& +\theta(z) g(x, J y)+\theta(J y) g(x, z)+\theta(J z) g(x, y)]\}, \\
& \left(\nabla_{x}^{\prime} \widetilde{g}\right)(y, z)=-Q(x, y, J z)-Q(x, J z, y) .
\end{aligned}
$$

Then, by (3.6) we get the following

Theorem 3.3. The complex connections $\nabla^{\prime}$ defined by (3.1) and (3.2) are natural on a $\mathcal{W}_{1}$-manifold if and only if $\lambda_{1}=\lambda_{2}=\lambda_{3}=\lambda_{4}=0, \lambda_{7}=-\lambda_{5}, \lambda_{8}=-\lambda_{6}$.

If we put $\lambda_{8}=-\lambda_{6}=s, \lambda_{7}=-\lambda_{5}=t, \lambda_{i}=0, i=1,2,3,4$, in (3.2), we obtain a two-parametric family of natural connections $\nabla^{\prime \prime \prime}$ defined by

$$
\begin{aligned}
\nabla_{x}^{\prime \prime \prime} y & =\nabla_{x} y+\frac{1-2 s}{2 n}[\theta(J y) x-g(x, y) J \Omega]+\frac{1}{n}\{s[g(x, J y) \Omega-\theta(y) J x] \\
& +t[g(x, y) \Omega-g(x, J y) J \Omega-\theta(y) x+\theta(J y) J x]\} .
\end{aligned}
$$

The well-known canonical connection [2] (or $B$-connection 3]) on a $\mathcal{W}_{1}$-manifold with Norden metric is obtained from (3.7) for $s=\frac{1}{4}, t=0$.

We give a summery of the obtained results in the following table

\begin{tabular}{|l|c|c|}
\hline Connection type & Symbol & $\lambda_{i} \in \mathbb{R}, i=1,2, \ldots, 8$. \\
\hline Complex & $\nabla^{\prime}$ & $\mu_{i}, i=1,2,3,4$, \\
\hline Complex & & $\nabla_{4}=\lambda_{5}, \mu_{2}=\lambda_{2}=\lambda_{6}=\lambda_{3}-\frac{1}{2}$, \\
symmetric & $\nabla^{\prime \prime}$ & $\mu_{1}=\lambda_{7}, \mu_{4}=\lambda_{8}$ \\
\hline & & $s, t$, \\
Natural & $\nabla^{\prime \prime \prime}$ & $s=\lambda_{8}=-\lambda_{6}, t=\lambda_{7}=-\lambda_{5}$, \\
& & $\lambda_{i}=0, i=1,2,3,4$. \\
\hline
\end{tabular}

Our next aim is to study the curvature properties of the complex connections $\nabla^{\prime}$. Let us first consider the natural connection $\nabla^{0}$ obtained from (3.7) for $s=t=0$, i.e.

$$
\nabla_{x}^{0} y=\nabla_{x} y+\frac{1}{2 n}[\theta(J y) x-g(x, y) J \Omega] .
$$

This connection is a semi-symmetric metric connection, i.e. a connection of the form $\nabla_{x} y+\omega(y) x-g(x, y) U$, where $\omega$ is a 1-form and $U$ is the corresponding vector of $\omega$, i.e. $\omega(x)=g(x, U)$. Semi-symmetric metric connections are introduced in [5] and studied in [6, 14]. The form of the curvature tensor of an arbitrary connection of this type is obtained in [14. The geometry of such connections on almost complex manifolds with Norden metric is considered in [10].

Let us denote by $R^{0}$ the curvature tensor of $\nabla^{0}$, i.e. $R^{0}(x, y) z=\nabla_{x}^{0} \nabla_{y}^{0} z-$ $\nabla_{y}^{0} \nabla_{x}^{0} z-\nabla_{[x, y]}^{0} z$. The corresponding tensor of type $(0,4)$ is defined by $R^{0}(x, y, z, u)=$ $g\left(R^{0}(x, y) z, u,\right)$. According to [14] it is valid 
Proposition 3.1. On a $\mathcal{W}_{1}$-manifold with closed 1-form $\theta^{*}$ the Kähler curvature tensor $R^{0}$ of $\nabla^{0}$ defined by (3.8) has the form

$$
R^{0}=R-\frac{1}{2 n} \psi_{1}(P),
$$

where

$$
P(x, y)=\left(\nabla_{x} \theta\right) J y+\frac{1}{2 n} \theta(x) \theta(y)+\frac{\theta(\Omega)}{4 n} g(x, y)+\frac{\theta(J \Omega)}{2 n} g(x, J y) .
$$

Since the Weyl tensor $W\left(\psi_{1}(S)\right)=0$ (where $S$ is a symmetric $(0,2)$-tensor), from (3.9) and (3.10) we conclude that

$$
W\left(R^{0}\right)=W(R) .
$$

Thus, the last equality implies

Proposition 3.2. Let $(M, J, g)$ be a $\mathcal{W}_{1}$-manifold with closed 1-form $\theta^{*}$, and $\nabla^{0}$ be the natural connection defined by (3.8). Then, the Weyl tensor is invariant by the transformation $\nabla \rightarrow \nabla^{0}$.

Further in this section we study the curvature properties of the complex connections $\nabla^{\prime}$ defined by (3.1) and (3.2). Let us denote by $R^{\prime}$ the curvature tensors corresponding to these connections.

If a linear connection $\nabla^{\prime}$ and the Levi-Civita connection $\nabla$ are related by an equation of the form (3.1), then, because of $\nabla g=0$, their curvature tensors of type $(0,4)$ satisfy

$$
\begin{aligned}
g\left(R^{\prime}(x, y) z, u\right) & =R(x, y, z, u)+\left(\nabla_{x} Q\right)(y, z, u)-\left(\nabla_{y} Q\right)(x, z, u) \\
& +Q(x, Q(y, z), u)-Q(y, Q(x, z), u),
\end{aligned}
$$

where $Q(x, y, z)=g(Q(x, y), z)$.

Then, by (3.1), (3.2), (3.9), (3.10), (3.12) we obtain the relation between $R^{\prime}$ and $R^{0}$ as follows

$$
\begin{aligned}
& R^{\prime}(x, y, z, u)=R^{0}(x, y, z, u)+g(y, z) A_{1}(x, u)-g(x, z) A_{1}(y, u) \\
& +g(x, u) A_{2}(y, z)-g(y, u) A_{2}(x, z)-g(y, J z) A_{1}(x, J u) \\
& +g(x, J z) A_{1}(y, J u)-g(x, J u) A_{2}(y, J z)+g(y, J u) A_{2}(x, J z) \\
& +\left[\frac{\lambda_{5} \lambda_{7}-\lambda_{6} \lambda_{8}}{n^{2}} \theta(\Omega)+\frac{\lambda_{7}-\lambda_{5}+2\left(\lambda_{5} \lambda_{8}+\lambda_{6} \lambda_{7}\right)}{2 n^{2}} \theta(J \Omega)\right]\left\{\pi_{1}-\pi_{2}\right\}(x, y, z, u) \\
& -\left[\frac{\lambda_{5} \lambda_{8}+\lambda_{6} \lambda_{7}}{n^{2}} \theta(\Omega)-\frac{\lambda_{6}-\lambda_{8}+2\left(\lambda_{5} \lambda_{7}-\lambda_{6} \lambda_{8}\right)}{2 n^{2}} \theta(J \Omega)\right] \pi_{3}(x, y, z, u),
\end{aligned}
$$

where

$$
\begin{aligned}
A_{1}(x, y) & =\frac{\lambda_{7}}{n}\left\{\left(\nabla_{x} \theta\right) y+\frac{\lambda_{7}}{n}[\theta(x) \theta(y)-\theta(J x) \theta(J y)]\right\} \\
& +\frac{\lambda_{8}}{n}\left\{\left(\nabla_{x} \theta\right) J y+\frac{1-2 \lambda_{8}}{2 n}[\theta(x) \theta(y)-\theta(J x) \theta(J y)]\right\} \\
& +\frac{\lambda_{7}\left(4 \lambda_{8}-1\right)}{2 n^{2}}[\theta(x) \theta(J y)+\theta(J x) \theta(y)], \\
A_{2}(x, y) & =-\frac{\lambda_{5}}{n}\left\{\left(\nabla_{x} \theta\right) y-\frac{\lambda_{5}}{n}[\theta(x) \theta(y)-\theta(J x) \theta(J y)]\right\} \\
& -\frac{\lambda_{6}}{n}\left\{\left(\nabla_{x} \theta\right) J y+\frac{1+2 \lambda_{6}}{2 n}[\theta(x) \theta(y)-\theta(J x) \theta(J y)]\right\} \\
& +\frac{\lambda_{5}\left(4 \lambda_{6}+1\right)}{2 n^{2}}[\theta(x) \theta(J y)+\theta(J x) \theta(y)] .
\end{aligned}
$$

We are interested in necessary and sufficient conditions for $R^{\prime}$ to be a Kähler curvature-like tensor, i.e. to satisfy (2.8) and (2.10). From (2.11), (3.13) and 
(3.14) it follows that $R^{\prime}$ is Kählerian if and only if $A_{1}(x, y)=A_{2}(x, y)$. Hence we obtain

Theorem 3.4. Let $(M, J, g)$ be a conformal Kähler manifold with Norden metric, and $\nabla^{\prime}$ be the complex connection defined by (3.1) and (3.2). Then, $R^{\prime}$ is a Kähler curvature-like tensor if and only if $\lambda_{7}=-\lambda_{5}$ and $\lambda_{8}=-\lambda_{6}$. In this case, from (3.1) and (3.2) we obtain a six-parametric family of complex connections $\nabla^{\prime}$ whose curvature tensors $R^{\prime}$ have the form

$$
\begin{aligned}
R^{\prime} & =R^{0}+\frac{\lambda_{7}}{n}\left\{\psi_{1}-\psi_{2}\right\}\left(S_{1}\right)+\frac{\lambda_{8}}{n}\left\{\psi_{1}-\psi_{2}\right\}\left(S_{2}\right) \\
& +\frac{\lambda_{7}\left(4 \lambda_{8}-1\right)}{2 n^{2}}\left\{\psi_{1}-\psi_{2}\right\}\left(S_{3}\right)+\frac{\lambda_{7}\left(1-2 \lambda_{8}\right) \theta(J \Omega)}{n^{2}}\left\{\pi_{1}-\pi_{2}\right\} \\
& +\frac{2 \lambda_{7} \lambda_{8} \theta(\Omega)}{n^{2}} \pi_{3},
\end{aligned}
$$

where $R^{0}$ is given by (3.9), (3.10), and

$$
\begin{aligned}
S_{1}(x, y) & =\left(\nabla_{x} \theta\right) y+\frac{\lambda_{7}}{n}[\theta(x) \theta(y)-\theta(J x) \theta(J y)]-\frac{\lambda_{7} \theta(\Omega)}{2 n} g(x, y) \\
& +\frac{\lambda_{7} \theta(J \Omega)}{2 n} g(x, J y), \\
S_{2}(x, y) & =\left(\nabla_{x} \theta\right) J y+\frac{1-2 \lambda_{8}}{2 n}[\theta(x) \theta(y)-\theta(J x) \theta(J y)] \\
& +\frac{\lambda_{8} \theta(\Omega)}{2 n} g(x, y)+\frac{\left(1-\lambda_{8}\right) \theta(J \Omega)}{2 n} g(x, J y), \\
S_{3}(x, y) & =\theta(x) \theta(J y)+\theta(J x) \theta(y) .
\end{aligned}
$$

From (3.15), (3.16) and Theorem 3.4 we get

Corollary 3.1. Let $(M, J, g)$ be a conformal Kähler manifold with Norden metric and $\nabla^{\prime}$ be the eight-parametric family of complex connections defined by (3.1) and (3.2). Then $R^{\prime}=R^{0}$ if and only if $\lambda_{i}=0$ for $i=5,6,7,8$.

Let us remark that by putting $\lambda_{i}=0$ for $i=1,2,5,6,7,8$ in (3.2) we obtain a two-parametric family of complex connections whose Kähler curvature tensors coincides with $R^{0}$ on a $\mathcal{W}_{1}$-manifold with closed 1 -form $\theta^{*}$.

Theorem 3.2 and Corollary 3.1 imply

Corollary 3.2. On a conformal Kähler manifold with Norden metric the Weyl tensor is invariant by the transformation of the Levi-Civita connection in any of the complex connection $\nabla^{\prime}$ defined by (3.1) and (3.2) for $\lambda_{i}=0, i=5,6,7,8$.

Since for the Bochner tensor of $\left\{\psi_{1}-\psi_{2}\right\}(S)$ it is valid $B\left(\left\{\psi_{1}-\psi_{2}\right\}(S)\right)=0$, where $S$ is symmetric and hybrid with respect to $J$, from Theorem 3.4 and (2.11) it follows

$$
B\left(R^{\prime}\right)=B\left(R^{0}\right) .
$$

By this way we proved the truthfulness of the following

Theorem 3.5. Let $(M, J, g)$ be a conformal Kähler manifold with Norden metric, $R^{\prime}$ be the curvature tensor of $\nabla^{\prime}$ defined by (3.1) and (3.2) for $\lambda_{7}=-\lambda_{5}, \lambda_{8}=-\lambda_{6}$, and $R^{0}$ be the curvature tensor of $\nabla^{0}$ given by (3.8). Then the Bochner tensor is invariant by the transformations $\nabla^{0} \rightarrow \nabla^{\prime}$.

\section{Conformal transformations of COMPlex CONnECTIONS}

In this section we study usual conformal transformations of the complex connections $\nabla^{\prime}$ defined in the previous section. 
Let $(M, J, g)$ and $(M, J, \bar{g})$ be conformally equivalent almost complex manifolds with Norden metric by the transformation (2.12). It is known that the Levi-Civita connections $\nabla$ and $\bar{\nabla}$ of $g$ and $\bar{g}$, respectively, are related as follows

$$
\bar{\nabla}_{x} y=\nabla_{x} y+\sigma(x) y+\sigma(y) x-g(x, y) \Theta,
$$

where $\sigma(x)=d u(x)$ and $\Theta=\operatorname{grad} \sigma$, i.e. $\sigma(x)=g(x, \Theta)$. Let $\bar{\theta}$ be the Lie 1-form of $(M, J, \bar{g})$. Then by (2.4) and (4.1) we obtain

$$
\bar{\theta}=\theta+2 n(\sigma \circ J), \quad \bar{\Omega}=e^{-2 u}(\Omega+2 n J \Theta) .
$$

It is valid the following

Lemma 4.1. Let $(M, J, g)$ be an almost complex manifold with Norden metric and $(M, J, \bar{g})$ be its conformally equivalent manifold by the transformation (2.12). Then, the curvature tensors $R$ and $\bar{R}$ of $\nabla$ and $\bar{\nabla}$, respectively, are related as follows

$$
\bar{R}=e^{2 u}\left\{R-\psi_{1}(V)-\pi_{1} \sigma(\Theta)\right\}
$$

where $V(x, y)=\left(\nabla_{x} \sigma\right) y-\sigma(x) \sigma(y)$.

Let us first study the conformal group of the natural connection $\nabla^{0}$ given by (3.8). Equalities (3.8) and (4.1) imply that its conformal group is defined analytically by

$$
\bar{\nabla}_{x}^{0} y=\nabla_{x}^{0} y+\sigma(x) y .
$$

It is known that if two linear connections are related by an equation of the form (4.4), where $\sigma$ is a 1 -form, then the curvature tensors of these connections coincide iff $\sigma$ is closed. Hence, it is valid

Theorem 4.1. Let $(M, J, g)$ be a $\mathcal{W}_{1}$-manifold with closed 1-form $\theta^{*}$. Then the curvature tensor $R^{0}$ of $\nabla^{0}$ is conformally invariant, i.e.

$$
\bar{R}^{0}=e^{2 u} R^{0} .
$$

Further in this section let $(M, J, g)$ be a conformal Kähler manifold with Norden metric. Then $(M, J, \bar{g})$ is a Kähler manifold and thus $\bar{\theta}=0$. From (4.2) it follows $\sigma=\frac{1}{2 n}(\theta \circ J)$. Then, from (3.1) and (3.2) we get $\bar{\nabla}^{\prime}=\bar{\nabla}$ and hence $\bar{R}^{\prime}=\bar{R}$ for all $\lambda_{i} \in \mathbb{R}, i=1,2, \ldots, 8$. In particular, $\bar{R}^{\prime}=\bar{R}^{0}$. Then, Theorems 3.5 and (3.17) imply

Theorem 4.2. On a conformal Kähler manifold with Norden metric the Bochner curvature tensor of the complex connections $\nabla^{\prime}$ defined by (3.1) and (3.2) with the conditions $\lambda_{7}=-\lambda_{5}$ and $\lambda_{8}=-\lambda_{6}$ is conformally invariant by the transformation (2.12), i.e.

$$
B\left(\bar{R}^{\prime}\right)=e^{2 u} B\left(R^{\prime}\right) .
$$

Let us remark that the conformal invariancy of the Bochner tensor of the canonical connection on a conformal Kähler manifold with Norden metric is proved in [3.

From Theorem 4.1 and Corollary 3.1 we obtain

Corollary 4.1. Let $(M, J, g)$ be a conformal Kähler manifold with Norden metric and $\nabla^{\prime}$ be a complex connection defined by (3.1) and (3.2). If $\lambda_{i}=0$ for $i=5,6,7,8$, then the curvature tensor of $\nabla^{\prime}$ is conformally invariant by the transformation (2.12). 


\section{An Example}

Let $G$ be a real connected four-dimensional Lie group, and $\mathfrak{g}$ be its corresponding Lie algebra. If $\left\{e_{1}, e_{2}, e_{3}, e_{4}\right\}$ is a basis of $\mathfrak{g}$, we equip $G$ with a left-invariant almost complex structure $J$ by

$$
J e_{1}=e_{3}, \quad J e_{2}=e_{4}, \quad J e_{3}=-e_{1}, \quad J e_{4}=-e_{2} .
$$

We also define a left-invariant pseudo-Riemannian metric $g$ on $G$ by

$$
\begin{aligned}
& g\left(e_{1}, e_{1}\right)=g\left(e_{2}, e_{2}\right)=-g\left(e_{3}, e_{3}\right)=-g\left(e_{4}, e_{4}\right)=1, \\
& g\left(e_{i}, e_{j}\right)=0, \quad i \neq j, \quad i, j=1,2,3,4 .
\end{aligned}
$$

Then, because of (2.1), (5.1) and (5.2), $(G, J, g)$ is an almost complex manifold with Norden metric.

Further, let the Lie algebra $\mathfrak{g}$ be defined by the following commutator relations

$$
\begin{aligned}
& {\left[e_{1}, e_{2}\right]=\left[e_{3}, e_{4}\right]=0,} \\
& {\left[e_{1}, e_{4}\right]=\left[e_{2}, e_{3}\right]=\lambda\left(e_{1}+e_{4}\right)+\mu\left(e_{2}-e_{3}\right),} \\
& {\left[e_{1}, e_{3}\right]=-\left[e_{2}, e_{4}\right]=\mu\left(e_{1}+e_{4}\right)-\lambda\left(e_{2}-e_{3}\right),}
\end{aligned}
$$

where $\lambda, \mu \in \mathbb{R}$.

The well-known Koszul's formula for the Levi-Civita connection of $g$ on $G$, i.e. the equality

$$
2 g\left(\nabla_{e_{i}} e_{j}, e_{k}\right)=g\left(\left[e_{i}, e_{j}\right], e_{k}\right)+g\left(\left[e_{k}, e_{i}\right], e_{j}\right)+g\left(\left[e_{k}, e_{j}\right], e_{i}\right),
$$

and (5.2) imply the following essential non-zero components of the Levi-Civita connection:

$$
\begin{array}{llrl}
\nabla_{e_{1}} e_{1} & =\nabla_{e_{2}} e_{2}=\mu e_{3}+\lambda e_{4}, & \nabla_{e_{3} e_{3}}=\nabla_{e_{4}} e_{4}=-\lambda e_{1}+\mu e_{2}, \\
\nabla_{e_{1}} e_{3}=\mu\left(e_{1}+e_{4}\right), & \nabla_{e_{1}} e_{4}=\lambda e_{1}-\mu e_{3}, \\
\nabla_{e_{2}} e_{3}=\mu e_{1}+\lambda e_{4}, & \nabla_{e_{2}} e_{4}=\lambda\left(e_{2}-e_{3}\right) .
\end{array}
$$

Then, by (2.2), (2.3) and (5.5) we compute the following essential non-zero components $F_{i j k}=F\left(e_{i}, e_{j}, e_{k}\right)$ of $F$ :

$$
\begin{aligned}
& F_{111}=F_{422}=2 \mu, \quad F_{222}=-F_{311}=2 \lambda, \\
& F_{112}=-F_{214}=F_{314}=-F_{412}=\lambda, \quad F_{212}=-F_{114}=F_{312}=-F_{414}=\mu .
\end{aligned}
$$

Having in mind (2.4) and (5.6), the components $\theta_{i}=\theta\left(e_{i}\right)$ and $\theta_{i}^{*}=\theta^{*}\left(e_{i}\right)$ of the 1 -forms $\theta$ and $\theta^{*}$, respectively, are:

$$
\theta_{2}=\theta_{3}=\theta_{1}^{*}=-\theta_{4}^{*}=4 \lambda, \quad \theta_{1}=-\theta_{4}=-\theta_{2}^{*}=-\theta_{3}^{*}=4 \mu .
$$

By (2.4) and (5.7) we compute

$$
\begin{gathered}
\Omega=4 \mu\left(e_{1}+e_{4}\right)+4 \lambda\left(e_{2}-e_{3}\right), \quad J \Omega=4 \lambda\left(e_{1}+e_{4}\right)-4 \mu\left(e_{2}-e_{3}\right), \\
\theta(\Omega)=\theta(J \Omega)=0 .
\end{gathered}
$$

By the characteristic condition (2.5) and equalities (5.6), (5.7) we prove that the manifold $(G, J, g)$ with Lie algebra $\mathfrak{g}$ defined by (5.3) belongs to the basic class $\mathcal{W}_{1}$. Moreover, by (5.5) and (5.7) it follows that the conditions (2.13) hold and thus

Proposition 5.1. The manifold $(G, J, g)$ defined by (5.1), (5.2) and (5.3) is a conformal Kähler manifold with Norden metric. 
According to (3.8), (5.2), (5.5) and (5.7) the components of the natural connection $\nabla^{0}$ are given by

$$
\begin{array}{lll}
\nabla_{e_{1}}^{0} e_{1}=-\nabla_{e_{4}}^{0} e_{1}=\mu e_{2}, & \nabla_{e_{2}}^{0} e_{1}=\nabla_{e_{3}}^{0} e_{1}=\lambda e_{2}, \\
\nabla_{e_{1}}^{0} e_{2}=-\nabla_{e_{4}}^{0} e_{2}=-\mu e_{1}, & \nabla_{e_{2}}^{0} e_{2}=\nabla_{e_{3}}^{0} e_{2}=-\lambda e_{1}, \\
\nabla_{e_{1}}^{0} e_{3}=-\nabla_{e_{4}}^{0} e_{3}=\mu e_{4}, & \nabla_{e_{2}}^{0} e_{3}=\nabla_{e_{3}}^{0} e_{3}=\lambda e_{4}, \\
\nabla_{e_{1}}^{0} e_{4}=-\nabla_{e_{4}}^{0} e_{4}=-\mu e_{3}, & \nabla_{e_{2}}^{0} e_{4}=\nabla_{e_{4}}^{0} e_{4}=-\lambda e_{3} .
\end{array}
$$

By (5.9) we obtain $R^{0}=0$. Then, by (3.9) and (3.10) the curvature tensor $R$ of $(G, J, g)$ has the form

$$
R=\frac{1}{4} \psi_{1}(A), \quad A(x, y)=\left(\nabla_{x} \theta\right) J y+\frac{1}{4} \theta(x) \theta(y) .
$$

Moreover, having in mind (3.16), (5.5), (5.7) and (5.8), we compute $S_{1}=S_{2}=$ $S_{3}=0$. Hence, for the tensors $R^{\prime}$ of the complex connections $\nabla^{\prime}$ given by (3.15), it is valid $R^{\prime}=0$.

Proposition 5.2. The complex connections $\nabla^{\prime}$ defined by (3.1) and (3.2) are flat on $(G, J, g)$.

\title{
REFERENCES
}

[1] G. Ganchev, A. Borisov, Note on the almost complex manifolds with a Norden metric, Compt. Rend. Acad. Bulg. Sci. 39(5) (1986), 31-34.

[2] G. Ganchev, V. Mihova, Canonical connection and the canonical conformal group on an almost complex manifold with B-metric, Ann. Univ. Sofia Fac. Math. Inform., 81(1) (1987), 195-206.

[3] G. Ganchev, K. Gribachev, V. Mihova, B-connections and their conformal invariants on conformally Kähler manifolds with B-metric, Publ. Inst. Math. (Beograd) (N.S.) 42(56) (1987), 107-121.

[4] K. Gribachev, D. Mekerov, G. Djelepov, Generalized B-manifolds, Compt. Rend. Acad. Bulg. Sci. 38(3) (1985), 299-302.

[5] A. Hayden, Subspaces of a space with torsion, Proc. London Math. Soc. 34 (1932), 27-50.

[6] T. Imai, Notes on semi-symmetric metric connections, Tensor N.S. 24 (1972), 293-296.

[7] S. Kobayshi, K. Nomizu, Foundations of differential geometry vol. 1, 2, Intersc. Publ., New York, 1963, 1969.

[8] A. Newlander, L. Nirenberg, Complex analytic coordinates in almost complex manifolds, Ann. Math. 65 (1957), 391-404.

[9] A. P. Norden, On a class of four-dimensional A-spaces, Russian Math. (Izv VUZ) 17(4) (1960), 145-157.

[10] S. D. Singh, A. K. Pandey, Semi-symmetric metric connections in an almost Norden metric manifold, Acta Cienc. Indica Math. 27(1) (2001), 43-54.

[11] M. Teofilova, Almost complex connections on almost complex manifolds with Norden metric, In: Trends in Differential Geometry, Complex Analysis and Mathematical Physics, eds. K. Sekigawa, V. Gerdjikov and S. Dimiev, World Sci. Publ., Singapore (2009), 231-240.

[12] K. Yano, Affine connections in an almost product space, Kodai Math. Semin. Rep. 11(1) (1959), 1-24.

[13] K. Yano, Differential geometry on complex and almost complex spaces, Pure and Applied Math. vol. 49, Pergamon Press Book, New York, 1965.

[14] K. Yano, On semi-symmetric metric connection, Rev. Roumanie Math. Pure Appl. 15 (1970), $1579-1586$.

\author{
Marta Teofilova \\ Department of Geometry \\ Faculty of Mathematics and Informatics \\ University of Plovdiv
}


COMPLEX CONNECTIONS ON CONFORMAL KÄHLER MANIFOLDS WITH NORDEN METRIG

236 Bulgaria Blvd.

4003 Plovdiv, Bulgaria

e-mail: marta@uni-plovdiv.bg 